\title{
Una aproximación a las relaciones entre espacio urbano y riesgo de desastres. Dos casos de estudio para el centro y la periferia inundable del Aglomerado Gran Buenos Aires ${ }^{1}$
}

\author{
Diego Ríos* \\ Silvia González**
}

\begin{abstract}
Resumen
A menudo los estudios que abordan la temática de riesgo de desastres le otorgan escasa importancia a la base urbana partícipe en la gestación de esos problemas. Este artículo propone una aproximación a las relaciones existentes entre espacio urbano y riesgo de desastres en términos de su producción, tomando dos estudios de caso para el centro y la periferia inundable del Aglomerado Gran Buenos Aires. Para ello se analizan los procesos materiales y simbólicos que participan de la incorporación urbana de áreas inundables (valle inferior del Arroyo Maldonado y bañados de Tigre), destacando la dinámica que adquiere el accionar de los actores económicos privados (asociados al capital inmobiliario-financiero) y el Estado durante los cambios ocurridos en las últimas décadas.
\end{abstract}

Palabras clave: espacio urbano; riesgo de desastres, áreas inundables; Buenos Aires; Tigre

\begin{abstract}
Studies addressing disaster risk issues normally give little importance to the urban aspects influencing the creation of these problems. This article proposes an approach to the relationship between urban space and disaster risks considering its production and taking into account two case studies -the centre and the floodable periphery of the Great Buenos Aires Agglomerations. It analyzes the material and symbolic processes involved in the urban incorporation of floodable areas (Maldonado Stream low basin and Tigre's marshes), highlighting the dynamics of the economic private actors (related to real state and financial capital) and the State during the changes of the last decades.
\end{abstract}

Key words: Urban Space; Disaster Risk, Flood-Prone Areas; Buenos Aires; Tigre

\section{Introducción}

Así como el riesgo caracteriza a la sociedad moderna, también la caracteriza su transformación en una sociedad primordialmente urbana (MANSILLA, 2000). Cada vez es mayor el porcentaje de población urbana respecto a la rural y esto implica un marcado crecimiento demográfico y espacial de las ciudades. En estos ámbitos se concentran bienes, infraestructura, población (con crecientes y variables niveles de vulnerabilidad social), lo que, sumado a una mayor exposición a fenómenos físicos extremos -y complejidadhace de las ciudades lugares favorables para la construcción de riesgos y la aparición de desastres.

Una de las principales causas de la ocurrencia de desastres en los ámbitos urbanos (por lo general minimizada o incluso excluida de

*Doctor en Geografía; investigador del Programa de Investigaciones sobre Recursos Naturales y Ambiente (PIRNA).e-mail: diegorios2005@yahoo.com.ar

** Doctora en Geografía; investigadora del Programa de Investigaciones sobre Recursos Naturales y Ambiente (PIRNA); e-mail: sgg@filo.uba.ar 
la explicación) radica en la urbanización de áreas donde acontecen fenómenos físicos extremos. Esto nos señala que la producción del espacio (como el urbano) adquiere un papel significativo en la comprensión de la ocurrencia y gestación de los desastres. En el espacio urbano los desastres se manifiestan aparentemente de forma "sorpresiva", cuando son en realidad el resultado "natural" de la forma en que es socializada la naturaleza. La fuerte presión que la sociedad ejerce sobre los recursos naturales, la transformación masiva de los ecosistemas locales o la incorporación de tecnologías y procesos productivos peligrosos, son algunos ejemplos de esa socialización.

El Aglomerado Gran Buenos Aires (AGBA), la mayor concentración urbana de la Argentina, es un ejemplo de lo dicho hasta aquí. Alrededor del $25 \%$ de su superficie se encuentra sobre áreas inundables (CLICHEVSKY, 2002), por lo que una parte significativa de su población y sus bienes son dañados por estos eventos. Dos de estas zonas inundables son el valle inferior del Arroyo Maldonado, en el área céntrica del AGBA y los bañados del Municipio de Tigre, en un área periférica del AGBA (ver Figura 1). A partir de la última década del siglo $X X$, momento de cambios sociales, políticos y económicos profundos en el país, el proceso de ocupación y valorización del espacio en ambas zonas ha adquirido características singulares en tanto que han sido protagonizados por grupos de ingresos medio-altos y altos, quebrando una vez más la idea preconcebida en la que se asocia a estos procesos sólo con los grupos más empobrecidos.

Figura 1.

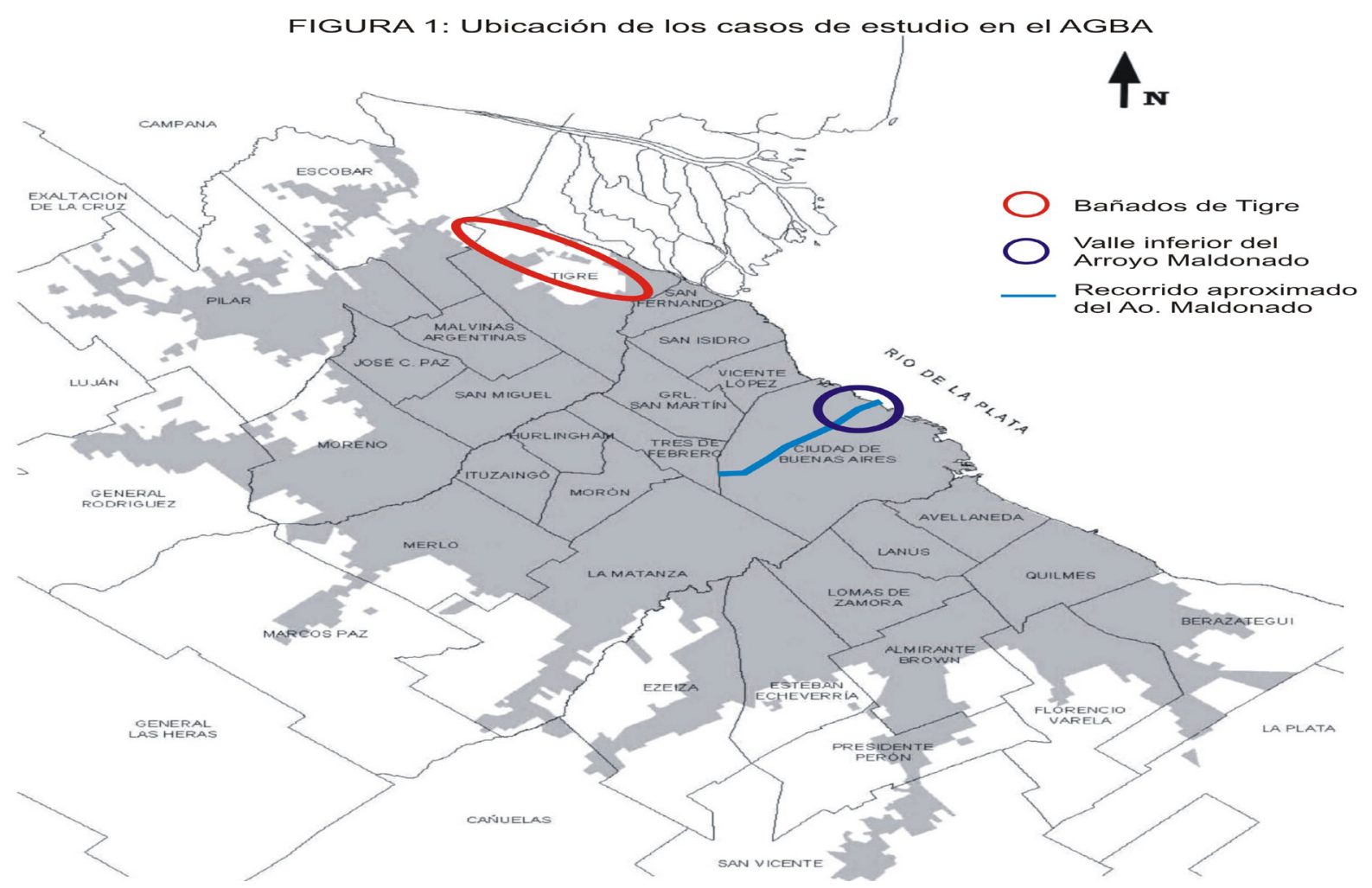


Entendemos que el estudio de riesgo de desastre en ámbitos urbanos debe superar los diagnósticos de aquello que está "expuesto"; entendiéndolo como suma de personas y bienes que se localizan en un espacio considerado como simple contenedor de cosas en un momento determinado. Por el contrario, consideramos que el espacio (en este caso el urbano) y su producción poseen un papel esencial en la comprensión de los riesgos de desastres. Es por ello que a continuación trabajaremos esta dimensión de la complejidad social a partir del análisis de los dos casos citados en la AGBA.

\section{Un punto de partida}

Es notable el avance en el conocimiento que las Ciencias Sociales han desarrollado en materia de riesgos y desastres durante las últimas décadas. Gracias a ello hoy en día es casi unánime la idea de que los desastres son una construcción social y que para su más correcta comprensión se debe focalizar en los procesos no evidentes de su constitución (la configuración del riesgo de desastre) que en la visibilidad de sus consecuencias (el desastre mismo). Ya casi nadie duda que el riesgo de desastre es un proceso socialmente dinámico que se produce históricamente (GARCÍA ACOSTA, 2005).

En este marco general, planteamos como punto de partida entender que los riesgos (como potencialidad) y los desastres (como su concreción) son resultado de las formas en que se desenvuelven y (re)producen las relaciones sociales. En esas relaciones el espacio juega un papel trascendente en tanto producto, medio y condición (CARLOS, 2008). Precisamente, poder entender la complejidad de dimensiones que participan de la producción material y simbólica del espacio realizado por la sociedad (y de las contradicciones resultantes que de ella surge) es uno de los caminos hacia la comprensión de la producción social de riesgo de desastres.

Siguiendo esta idea, cuando abordamos la producción de riesgos de desastres en la ciudad necesariamente debemos observar la lógica de la producción de espacio urbano en áreas donde ocurren fenómenos físicos extremos, tales como inundaciones, deslizamientos de tierra, derrames de petróleo, etc. En otras palabras, debemos analizar de qué forma son incorporadas ese tipo de áreas al espacio urbano -en el proceso de urbanización- y qué implicancias tiene dicha producción, en tanto creación de nuevos peligros (principalmente asociados al desarrollo tecnológico) o modificación de aquellos existentes. Al mismo tiempo la creciente situación de vulnerabilidad social, también cada vez más concentrada en los ámbitos urbanos, participa intensamente de dicha producción (MANSILLA, 2000). Por lo tanto, comprender la dinámica de la producción de espacio urbano en relación con los riesgos de desastres, implica entender cómo determinadas áreas donde ocurren fenómenos físicos extremos se pueden tornan peligrosas para los grupos que en ellas habitan dependiendo de sus condiciones de vulnerabilidad social.

Es por todo ello que nos interesa describir y analizar, como menciona Lindón (1989), la racionalidad con que la sociedad históricamente incorpora las áreas sujetas a fenómenos extremos al proceso de urbanización, en este caso particular, las áreas inundables del AGBA.

Por otro lado, la producción de espacio -en este caso, urbano- implica necesariamente un proceso de valorización, pues se trata del resultado de la relación sociedad-espacio, relación que está mediada por el trabajo humano. Tal como afirman Moraes y Da Costa (1987) la apropiación de recursos del espacio, la construcción de formas humanizadas sobre él, la conservación de esos constructos, las modificaciones sean del sustrato natural o de las obras realizadas por la sociedad, representan todas ellas una creación de valor. $A$ veces ciertas cualidades singulares, únicas o raras (sean naturales o construidas) del espacio son valorizadas por la sociedad. No obstante, por si solas esas cualidades no le otorgan un valor especial a determinado espacio (es decir, no son inherentes al mismo), sino que están asociadas a la valorización que de ellas hace la sociedad. En este sentido, Lindón (1989) sostiene que las cualidades del espacio no son estáticas, sino que se trata de componentes dinámicos modificables por la sociedad sujetos a patrones de valorización social, 
influidos por los niveles tecnológicos alcanzados y por los aspectos ideológico-culturales, variables en cada momento histórico.

Por ejemplo, la primera fundación de Buenos Aires, en 1536, se llevó a cabo sobre áreas inundables próximas a la desembocadura que el Riachuelo de los Navíos tenía sobre el Río de la Plata, siendo, entre otras, una de las condiciones que influyeron para que ese primer acto fundacional no resultara exitoso. El conocimiento que los españoles adquirieron en las primeras fundaciones fallidas en su empresa de conquista en América, derivó en el "decálogo de regulaciones urbanas" expresado en las Leyes de Indias de 1573 (HARDOY, 1989). Ya en la segunda fundación de Buenos Aires, en 1580, las tierras ubicadas sobre la barranca no sujetas a inundaciones fueron las valorizadas para su ocupación, dejando a las áreas inundables para aprovechamiento de materiales maderables -usados en construcción y como fuente de energía-, como sitios favorables para el desarrollo portuario, entre otros usos y finalidades. Más adelante en el tiempo, a medidos del siglo XIX, la inversión pública concretada en obras de consumo colectivo (afirmado y empedrado, creación de espacios de uso recreativo, etc.) valorizaron algunas de estas zonas inundables -como la cuenca del arroyo Tercero del Medio y el bajo del Retiro- y cambiaron entonces su uso social (LINDÓN, 1989). En este caso tuvo más peso específico el rédito económico privado en términos inmobiliarios que podía obtenerse de tal valorización -producida públicamente- que las condiciones negativas de inundabilidad.

En el proceso de valorización y producción del espacio urbano es significativo conocer qué actores sociales intervienen y con qué intereses, y a través de qué prácticas y discursos legitiman e incorporan las áreas donde acontecen fenómenos físicos extremos a la ciudad. En el ejemplo anterior hemos mencionado uno de esos actores, el Estado, que es clave no sólo por su papel de garante en la producción de espacio (MANSILLA, 2000), sino además por su papel en la producción de riesgos de desastres (STEINBERG, 2001).

Es el Estado, junto a los actores económicos privados, los que, a través de sus ideas, representaciones e imágenes, cumplen un papel privilegiado en la producción de discursos sobre el espacio urbano, lo cual legitima los procesos de valorización y transformación de determinados espacios, sean estos potencialmente peligrosos o no, en beneficio propio. Tales ideas y representaciones se expresan a través de prácticas y de discursos. Las prácticas, por ejemplo, pueden materializarse en obras públicas de consumo colectivo (Lindón, 1989), mientras que los discursos trasuntan las miradas dominantes sobre ese tipo singular de áreas; por ejemplo, si se tiende a cargar de significado positivo una zona inundable por su valor estético o si se tiende a negar el peligro a fin de validar la apropiación privada del espacio (STEINBERG, 2001).

En esta oportunidad, de las prácticas y discursos nos centraremos en las obras de infraestructura hidráulicas y en los discursos llevados a cabo/elaborados por los actores económicos privados y por el Estado, en tanto actores sociales con mayor decisión y poder en la producción del espacio urbano y, consecuentemente, en la producción social de riesgos de desastre por inundación en la AGBA.

\section{Los casos de estudio}

\section{I. El valle inferior del Arroyo Maldonado en la Ciudad Autónoma de Buenos Aires}

El Maldonado es uno de los antiguos arroyos porteños que fueron canalizados a principios del siglo XX. Ingresa a la Ciudad de Buenos Aires a la altura del barrio de Liniers, atraviesa el territorio porteño en sentido aproximado SO-NE y desemboca en el río de la Plata. Fue el antiguo límite de la ciudad entre 1867 y 1887 , año en el que se incorporaron los partidos de Flores y Belgrano a la capital nacional. Actualmente, la avenida Juan B. Justo corre sobre el techo de su canalización.

Como el resto de las áreas bajas de Buenos Aires, los humedales que rodeaban a su desembocadura no fueron valorizados en un primer momento para el asentamiento de población. El primer gran hito en la producción de espacio en esta zona se registró hacia la década de 1830 , fecha en la que el entonces gobernador de la provincia de Buenos Aires, Juan Manuel de Rosas, construyó 
su palacio en el llamado "Bañado de Palermo", nombre con el que se conocía a los humedales. La construcción del palacio, hecha durante el bloqueo francés al país, buscó reafirmar la confianza en una resolución favorable del conflicto y, por otra parte, demostrar al pueblo que era posible vencer los obstáculos que ofrecía la naturaleza² (DIRECCIÓN GENERAL DE PATRIMONIO, 2004). Unas décadas más tarde, y luego de la caída del gobernador, estas tierras pasaron a valorizarse como espacios públicos; allí se levantó el actual Parque Tres de Febrero.

Entre fines del siglo XIX y principios del XX comenzaron a formarse los caseríos que más tarde se convertirían en los diferentes barrios atravesados por el arroyo, desde el Bañado hacia aguas arriba. En esa época las inundaciones provocadas por el desborde del arroyo Maldonado empezaron a preocupar seriamente a las autoridades locales y nacionales. Luego de algunas obras menores, Obras Sanitarias de la Nación proyectó la más importante intervención del Estado en la mitigación de inundaciones en la ciudad: el Plan General de Desagües Pluviales. Este Plan tuvo como objetivo proveer de desagües pluviales a todo el sector de la ciudad de Buenos Aires que carecía de ellos (el llamado "Radio Nuevo"), lo cual garantizaría tanto el saneamiento del territorio porteño como la desaparición de las inundaciones (González, 1997). Para lograr estos objetivos se aprovecharon los cursos de agua naturales aún existentes en la ciudad y se los transformó en los emisarios principales que conducirían toda el agua pluvial hacia el río de la Plata. El Plan resolvió de manera independiente el desagüe de cada una de las grandes cuencas (Maldonado, Medrano, Vega y Riachuelo), teniendo en cuenta las divisorias de aguas. Para el caso del Maldonado, se proyectó una canalización cerrada a la que llegaría el agua de lluvia a través de conductos secundarios, con lo que, además, se suprimió el "aspecto antiestético" de un canal a la vista atravesando la ciudad. La propuesta, además, incluía la construcción de una avenida, con un paseo central amplio en el que se planteaba el establecimiento de ferias y edificios para empleados u obreros (GONZÁLEZ, 1997).

Si bien en la práctica este ambicioso proyecto quedó reducido durante mucho tiempo a una calle de tierra, usada como basurero y refugio de "mal vivientes", es claro que además de la pretendida supresión de las inundaciones, el saneamiento del valle del Maldonado buscó incorporar nuevo suelo urbano a la ciudad y valorizarlo en el proceso. La construcción de la Av. Juan B. Justo sobre el techo de la canalización (hecha por tramos, entre 1937 y 1950) contribuyó a este proceso, al igual que la adopción de algunos instrumentos normativos tendientes a regular la producción de espacio urbano. La consecuencia inmediata fue una rápida urbanización de los barrios cruzados por el antiguo Maldonado, los que se transformaron en zonas de alta densidad poblacional (HERZER y CLICHEVSKY, 2001) hacia mediados del siglo XX.

Este proceso repercutió sobre la infraestructura diseñada para hacer "desaparecer" las inundaciones, que resultó insuficiente. Fue entonces que los puntos críticos que se inundaban en las primeras décadas del siglo $X X$ volvieron a inundarse cíclicamente. Sin embargo, recién con la gran inundación de mayo de 1985 se puso en escena el problema y se comenzó nuevamente a trabajar en su "solución". El Estado nacional y el municipal propusieron varios planes que en general buscaban aliviar la carga del emisario principal y/o retener aguas para retardar el escurrimiento. Ninguno de ellos se llevó a cabo, a excepción hecha de obras puntuales enmarcadas en el Plan de Control de Inundaciones de la Ciudad de Buenos Aires, tendientes a mejorar la capacidad de carga del Maldonado entubado ${ }^{3}$ (GONZÁLEZ, 2006), cosa que se logró en gran medida. A esa obra se sumó a fines de la década de 1990 la propuesta de construir otra de mayor envergadura en el marco del Plan Maestro de Ordenamiento Hidráulico, cuya ejecución está en marcha, habiéndose finalizado una parte en septiembre de 2010 .

Estas intervenciones del Estado han coincidido con un cambio sustancial en la producción del espacio urbano, impulsado por la particular coyuntura política económica que dominó la década de 1990 y que se sintetiza en un nuevo modelo de relación entre el Estado y el sector privado. Este nuevo modelo se expresó desde lo discursivo en la sanción de leyes paradigmáticas ${ }^{4}$ que dieron paso, en la acción, a un intenso proceso de privatización 
de servicios y tierras públicas, y de desregulación y flexibilización de las relaciones de mercado a todo nivel.

En la Ciudad de Buenos Aires se agregaron además algunas decisiones del gobierno local como las modificaciones introducidas en el Código de Planeamiento Urbano (CPU) -la principal normativa tendiente a regular la producción de espacio-, que siguiendo la lógica dominante del período, fue un paso necesario para garantizar la flexibilización del mercado inmobiliario y liberar la construcción en altura (CLICHEVSKY, 1996). Estas modificaciones (realizadas en los años 1989 y 2000) se agregaron a una práctica usual durante la década de 1990: la utilización del mecanismo de "excepción" a la norma para la construcción de torres y grandes edificios $^{5}$ (HERZER y CLICHEVSKY, 2001). Ambos hechos han impulsado un segundo período de verticalización de la ciudad ${ }^{6}$, que con la aparición de grandes inversores privados (los llamados "desarrolladores urbanos"), dieron lugar a procesos selectivos de producción del espacio justamente en aquellas áreas favorecidas por la intervención del Estado y por eso mismo atractivas para la gran inversión inmobiliaria.

De la mano de esos procesos se produjo un cambio en las preferencias de los consumidores de clase alta y media alta, ligada a tendencias mundiales en la búsqueda de un mayor confort en la vivienda. Esto dio lugar a la aparición de una nueva tipología constructiva (la "torre amurallada de alto estándar" o "torre country") en la que se combina la disponibilidad de servicios de alto nivel7, la seguridad, el valorado -aunque limitadocontacto con la naturaleza ${ }^{8}$ y una ubicación privilegiada en términos de accesibilidad al resto de la ciudad. Todo esto no hace sino reconfigurar las áreas donde se ubican las torres desde el punto de vista social y espacial (SZAJNBERG y CORDARA, 2005).

Una de las zonas que más ha crecido en virtud de estos procesos desde la década de los noventa es precisamente el valle inferior del arroyo Maldonado, aquel ocupado por el Bañado de Palermo y hoy asiento del barrio del mismo nombre. Este barrio y más precisamente algunos de sus sectores (conocidos como "Palermo Nuevo" y "Palermo Boulevard", ver Figura 2), fueron una de la zonas privilegiadas por los nuevos desarrolladores urbanos precisamente por su "ubicación estratégica en el corredor norte y su excelente accesibilidad" (SZAJNBERG y CORDARA, 2005). A estas razones deben agregarse, además, algunas inversiones a gran escala del Estado (nacional y local) que han valorizado enormemente el espacio. Se trata de grandes emprendimientos de diverso tipo y de proyectos que a futuro también tienden a la reconversión social y territorial ${ }^{9}$ y la recuperación de edificios de valor histórico en la

La consecuencia lógica de todos estos procesos es la re-densificación de un espacio crítico, sujeto aún a inundaciones periódicas. Esta aparente contradicción se apoya, desde lo discursivo, en la alta confianza de los desarrolladores, vecinos y hasta analistas del fenómeno "palermitano" en general en las obras ya realizadas en la canalización del Maldonado y los proyectos que se llevarían a cabo en un futuro próximo. Al respecto, es interesante marcar que estos actores señalan las obras -es decir, la inversión pública- como otra de las causas del "boom" inmobiliario en Palermo. La alta confianza en el saber experto -la ingenieríacomo garante de la "desaparición" de la inundación es reforzada además por una suerte de "negación" (STEINBERG, 2001) del problema sustentado en la ausencia de eventos severos desde el año 2001.

Este breve recorrido por la historia del valle inferior del Maldonado permite ilustrar de qué manera se articula la producción del espacio con la producción del riesgo. Desde la cuestión puramente política -que impulsa el relleno de bajíos como primer gran hito en el "dominio" de la peligrosidad-, hasta la lógica de los actores hegemónicos en las últimas décadas -que imponen sus intereses articulando el discurso (privatización, desregulación, consumo globalizado) con la acción pública como sostén del negocio privado-, se asiste a un proceso en el que el riesgo se construye y se reconstruye impulsado por modelos vigentes de producción y consumo del espacio urbano en cada etapa. En este proceso, el impacto de cada inundación se amplifica hasta alcanzar niveles que un futuro probable serán tan altos como las nuevas "torres country" de Palermo. 
Figura 2.

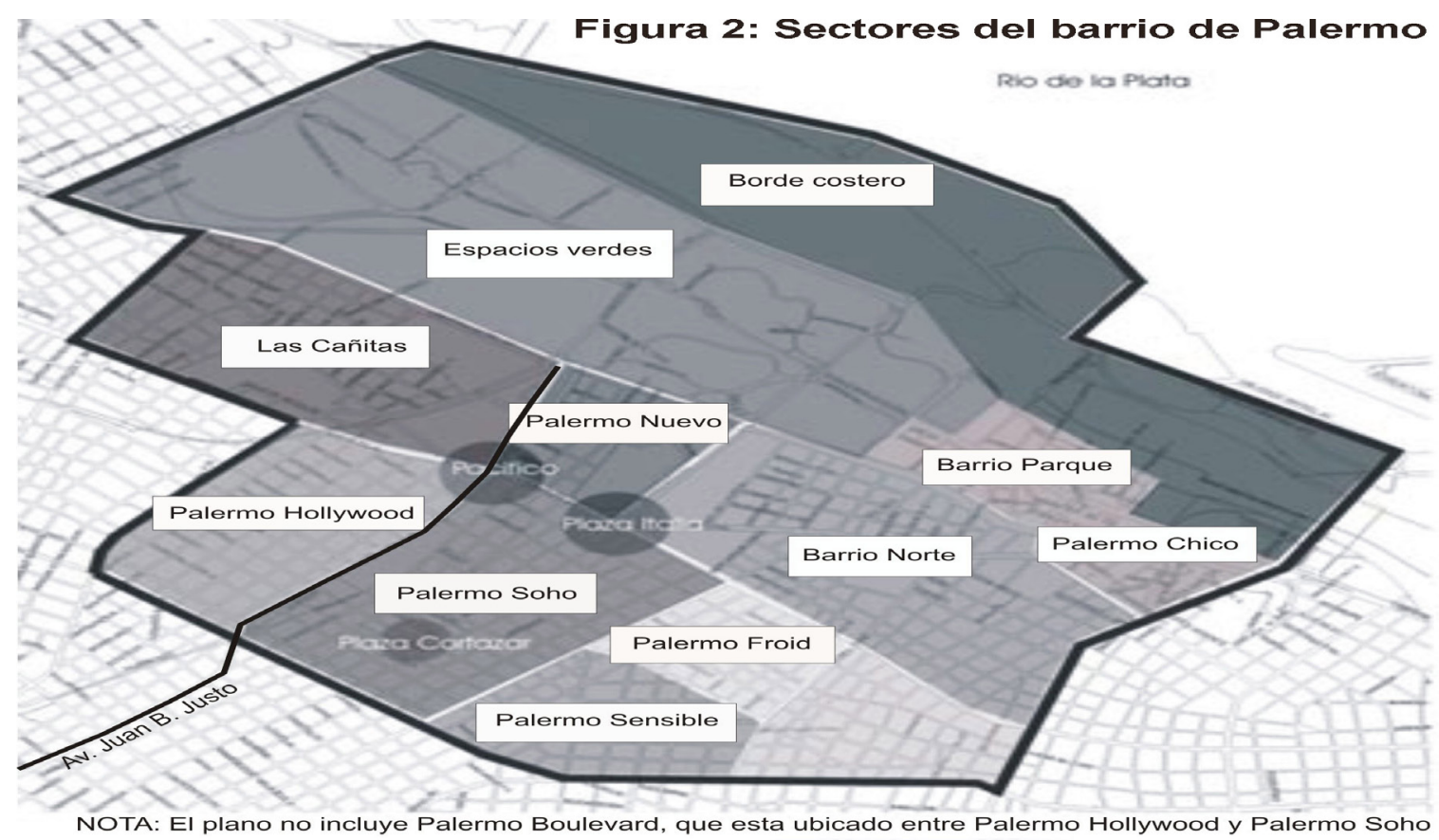

\section{III.II. Los bañados del Municipio de Tigre, Provincia de Buenos Aires}

Los bañados del municipio de Tigre se destacan como uno de los lugares de la periferia del AGBA donde la articulación entre producción de espacio urbano y de riesgo de desastres manifiesta uno de los más ricos pasados $y$, a su vez, un presente repleto de significativas transformaciones. A continuación, se detallan dos momentos en el que se aborda dicha articulación, en base a la valorización diferencial que se hace de ese espacio inundable: a) Los comienzos de su poblamiento y la importancia de sus condiciones de sitio y posición para la instalación de un puerto, y b) Las transformaciones inéditas en materia de urbanización de esas tierras inundables activadas por la expansión de urbanizaciones cerradas (UCS) sobre rellenos durante las últimas décadas.

Pocos años después de la segunda fundación de Buenos Aires, en la primera década del siglo XVII, empezó a formarse un paraje en la desembocadura que el río Las Conchas (actual Reconquista) tenía sobre el Río de la Plata. A pesar que eran tierras sumamente inundables, por lo cual no respetaba las recomendaciones indicadas en las Leyes de Indias (1573), la zona fue valorizada como adecuada por otra de sus condiciones de sitio propicias para la instalación de un puerto: protección de los navíos ante los efectos de las tormentas (fuertes vientos del sudeste) en el Río de la Plata y calado satisfactorio para la época. Por el precario puerto de Las Conchas se abastecía a los habitantes de Buenos Aires de recursos maderables (maderas, leñas y carbón) y otros productos provenientes de las islas del Delta y de Asunción del Paraguay. Ya para fines del siglo XVIII el puerto de Las Conchas y el caserío circundante fue adquiriendo mayor desarrollo comercial, especialmente, al constituirse en un nexo de importancia para las actividades de contrabando de mercancías ilícitas (en su mayoría 
de origen inglés) que se filtraban a los controles de la Metrópoli española, una vez fundado el puerto de Colonia do Sacramento por los portugueses en la banda opuesta del Río de la Plata (SILVA, 1989; UDAONDO, 2001).

Desde sus comienzos los pobladores de Las Conchas tuvieron que lidiar con las inundaciones, pero es recién a inicios del siglo XIX que este fenómeno empieza a ser registrado por la historiografía como generador de acontecimientos desastrosos de importancia. Una de las inundaciones más dañinas fue desatada por una sudestada muy enérgica ocurrida en junio de 1805 que hizo crecer bruscamente el nivel de las aguas, anegando todo el bañado hasta la base de las barrancas cercanas ${ }^{11}$. Frente a este desastre y ante la solicitud de un representante de la iglesia en nombre de los pobladores, el Gobierno del Virreinato dispuso el traslado de la población de esas tierras consideradas como peligrosas a un sitio en el alto: la Punta Gorda, donde se fundara en febrero de 1806 San Fernando de la Buenavista. Junto al nuevo pueblo se mandó crear un canal que sirviera de puerto y desagüe del río de Las Conchas (UDAONDO, 2001). Este hecho es uno de los primeros antecedentes de la intervención del Estado en el que se plantea como solución el traslado de la población y la construcción de obras de infraestructura. Es probable que el canal se haya pensado, por un lado, para disminuir las consecuencias dañinas de las inundaciones (a pesar de que la obra nunca se concluyó, más allá de la existencia de diversos proyectos que indicaban un interés en hacerlo) y, por otro, para asegurar el comercio de productos maderables $y$, especialmente, el ingreso de productos contrabandeados, a través de un nuevo puerto que beneficiaba a los comerciantes del flamante pueblo de San Fernando, en detrimento de los intereses de los de Las Conchas.

En agosto de 1820 se produjo otro desastre de grandes magnitudes originado por la conjunción de una creciente del río Las Conchas y una intensa sudestada, en el que perecieron más de noventa habitantes del poblado de Las Conchas. La fuerte corriente generada por la crecida no sólo destruyó gran parte del poblado y su iglesia, sino que además cambió la configuración espacial de la zona; al tiempo que quedó obstruida la entrada al puerto sobre el río Las Conchas, se agrandó y aumentó el calado del arroyo del Tigre, que hasta este entonces no se extendía por más de 600 metros tierra adentro. A partir de esa gran inundación el poblado resultó incomunicado quedando encerrado entre el río Las Conchas y el mencionado arroyo. Asimismo, las mejores condiciones que presentaba la desembocadura del arroyo del Tigre (como por ejemplo su mayor calado) hicieron que ese sitio se convirtiera en el nuevo puerto de pueblo de Las Conchas. De allí que el "puerto del Tigre" terminó dando su nombre a la ciudad y, más tarde en 1954, al Municipio. Según Udaondo (1942, p. 55), al año siguiente (1821) el Gobierno expidió un decreto en el que "se prohíbe que nadie pueda poblar o edificar en adelante ni en el pueblo de Las Conchas ni tampoco en los alrededores que están expuestos a inundaciones"; decisión que otra vez resultó quebrantada por la población. En ese momento, ante el pedido de los comerciantes del pueblo de San Fernando, el Gobierno provincial aceptó continuar con las obras del Canal homónimo (iniciado desde el evento anterior), en tanto que los vecinos y comerciantes de Las Conchas, solicitaron que se construya un puente sobre el arroyo Tigre y un veredón (terraplén) para comunicar a Las Conchas hasta el Canal en caso de inundaciones (utilizado unas décadas después para la construcción del Ferrocarril Mitre). La realización de este conjunto de obras públicas terminaron valorizando las tierras del bañado que quedaban entre el poblado de Las Conchas y el Canal San Fernando, formándose en poco tiempo un continuo urbano entre ambos pueblos.

Con el transcurrir del tiempo las tierras inundables de los bañados de Las Conchas/ Tigre fueron siendo incorporadas en distintos momentos $^{12}$, bajo racionalidades diferentes, al proceso de urbanización. El punto de quiebre más significativo en ese proceso (ver Figura 3 ) se desenvuelve a partir de la explosiva expansión de las UCs en la última década del siglo XX, y que continúa en la actualidad. Para 1990 la superficie ocupada por UCs era de 202 hectáreas mientras que a fines de 2009 esa superficie había alcanzado las 4.035 hectáreas (RÍOS, 2010). 


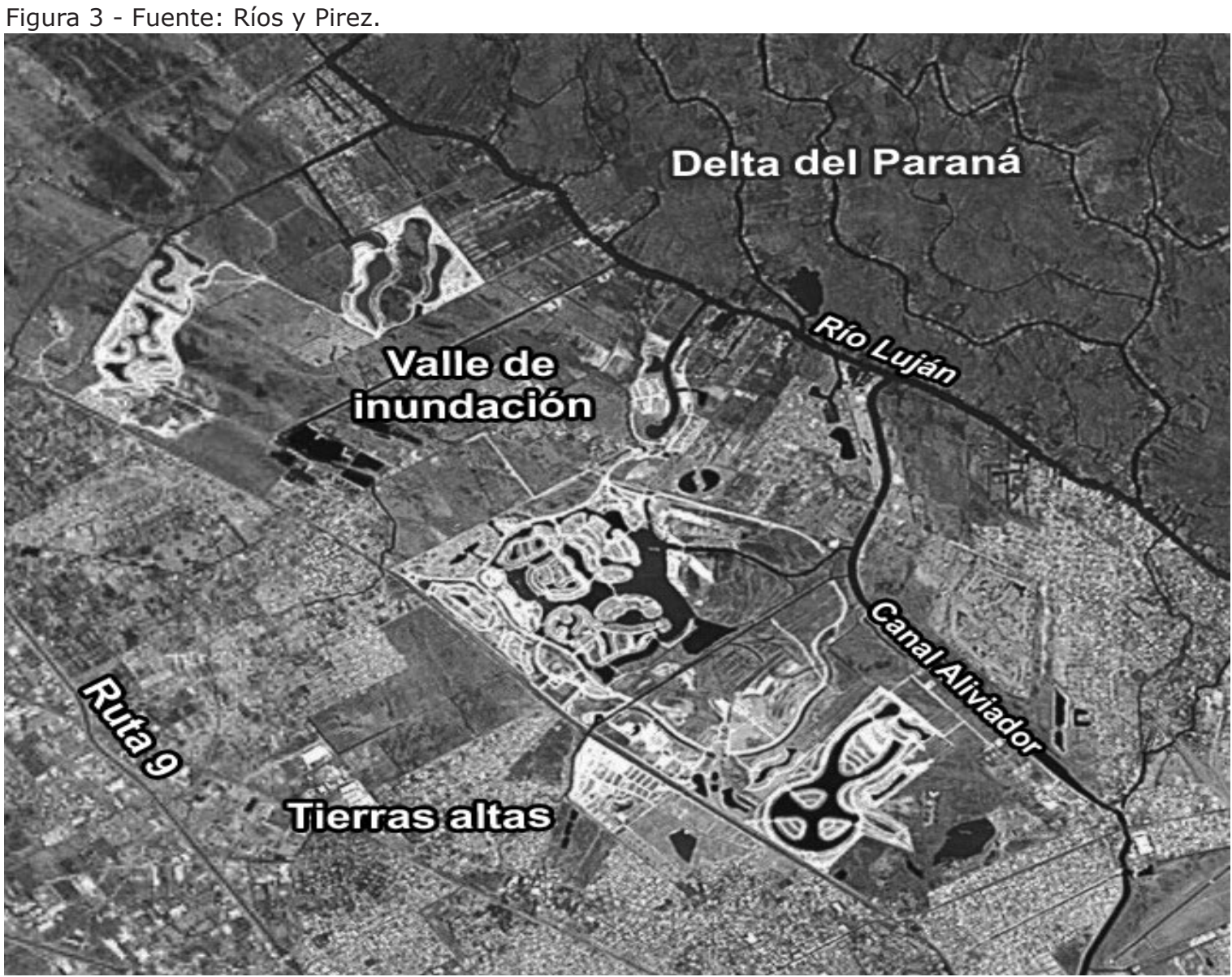

Dicha notable expansión se enmarca en la modificación de la articulación público-privado ocurrida en los años noventa en Argentina, vinculada a la disminución de las inversiones públicas, al apoyo estatal a la actividad privada y al papel protagónico de las inversiones privadas en la producción del espacio urbano; papel en el que la participación de capitales internacionales adquieren cada vez mayor peso en la producción de la ciudad $^{13}$. También debe entenderse esta expansión en un contexto donde las UCs se convierten en un importante "negocio urbano" tanto para los actores económicos privados, como para los propios gobiernos municipales que ven en ellas la posibilidad de alcanzar el tan ansiado "desarrollo urbano" en sus tierras vacantes. Para que se dé este proceso expansivo fueron necesarios no sólo los capitales privados, sino también la participación de fuertes inversiones públicas (en los distintos niveles, pero principalmente locales) en obras de consumo colectivo (obras en infraestructuras hidráulica y de saneamiento, como las del UNIREC ${ }^{14}$ u obras de embellecimiento urbano, como el Paseo Victorica, entre otras), como también cambios en la normativa de zonificación de uso del suelo (pasaje de uso rural a urbano a través de la Ordenanza no $1894 / 96$, por ejemplo), con el fin de maximizar las ganancias de los actores económicos privados y de atraer mayores inversiones privadas al Municipio (RÍOS y PIREZ, 2008). 
Figura 4: Nordelta. Construcción de rellenos a través de la técnica de movimiento de suelos y refulado hidráulico
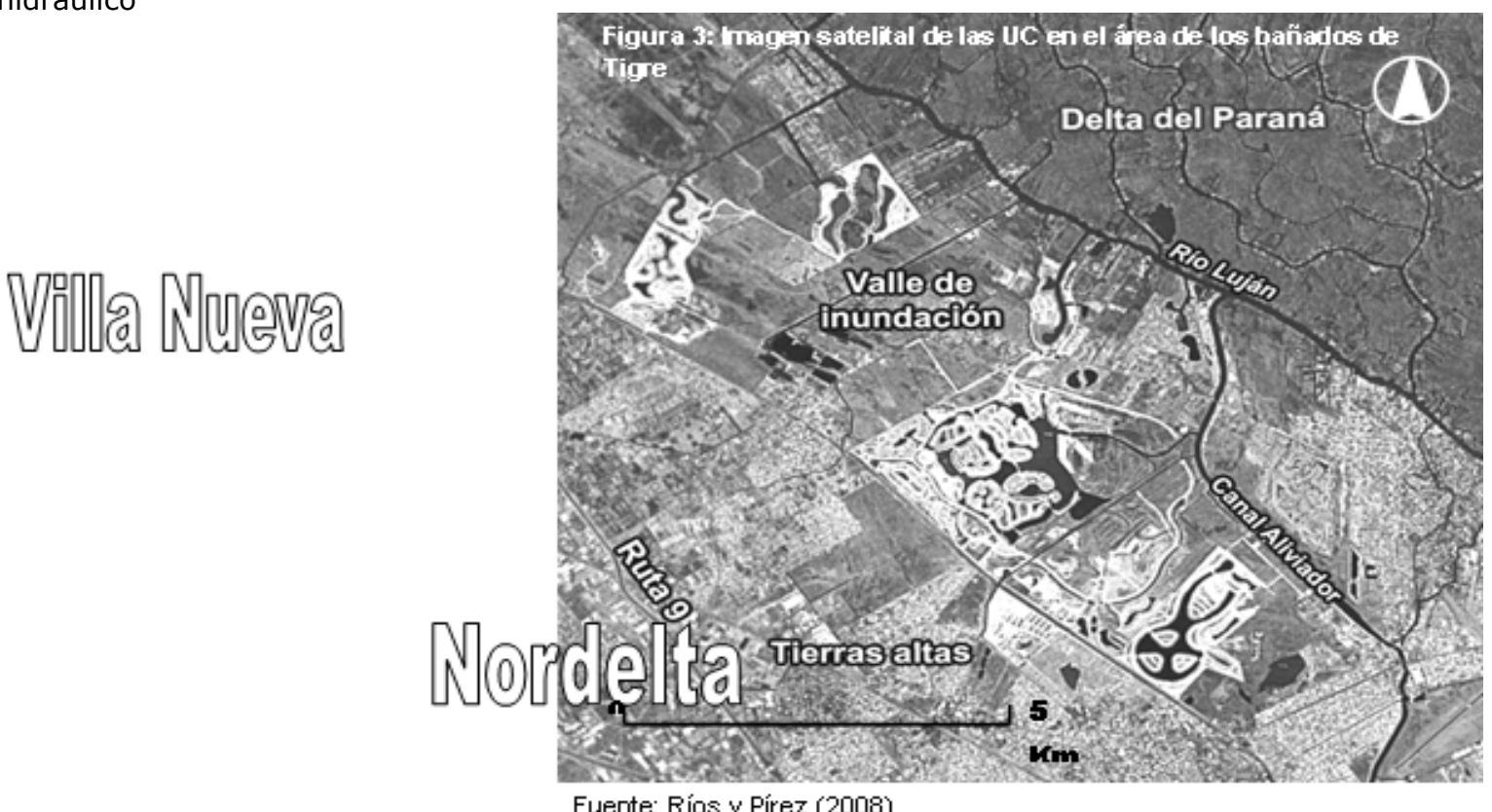

A lo largo del tiempo la sociedad le concedió escaso valor uso a la mayor parte de las tierras inundables de los bañados de Tigre por sus características de inundabilidad y anegabilidad, la alta salinidad de los suelos y nivel de la napa freática, como también su limitada accesibilidad, restringiendo cualquier tipo de actividad productiva. Precisamente, esas mismas características que le otorgaban un exiguo costo en el mercado de tierras, fueron valorizadas por los actores económicos privados desarrolladores de UCs como condición necesaria para desplegar un gran negocio urbano en el contexto de cambio de época que fueron los años noventa. La urbanización de los bañados previa a la expansión de las UCs estuvo limitada por las características mencionadas y también -en menor medida- por las restricciones legales para urbanizar sobre zonas inundables. Entre estas se destaca la Ley Provincial no 6254/60 (aún vigente) que prohíbe realizar fraccionamientos por debajo de cota mínima de piso de 3,75 m (Instituto Geográfico Nacional-IGN). En la actualidad los proyectos de UCs que quieren desarrollarse en la

periferia inundable del AGBA deben cumplir con ésta última ley al presentar el estudio de aptitud hídrica y el proyecto de obra de hidráulica en los organismos técnicos competentes de la Provincia de Buenos Aires.

Ahora bien, ¿cómo fueron superadas en el caso de las UCs las limitaciones físiconaturales y jurídicas que impedían transformar las tierras inundables en tierras urbanizables, dando lugar a un formidable negocio urbano? Con la incorporación de trabajo, grandes capitales y tecnologías especializadas (movimientos de suelos) se edificaron enormes rellenos (ver Figura $4)^{14}$, que implicaron el movimiento de más de 50 millones de metros cúbicos de tierra, permitiendo alcanzar la "cota de seguridad" requerida por las normas, elevando en promedio $1,7 \mathrm{~m}$ el nivel del terreno. De esta manera, dichos rellenos fueron el sistema constructivo utilizado para incorporar esas condiciones físico-naturales adversas al proceso de producción del espacio urbano $y$, al mismo tiempo, cumplir con la reglamentación vigente (RÍOS, 2010). 
Figura 5. Fuente: presentación de Nordelta SA. en $V$ Jornada de Desarrollos Suburbanos, septiembre de 2006, Buenos Aires

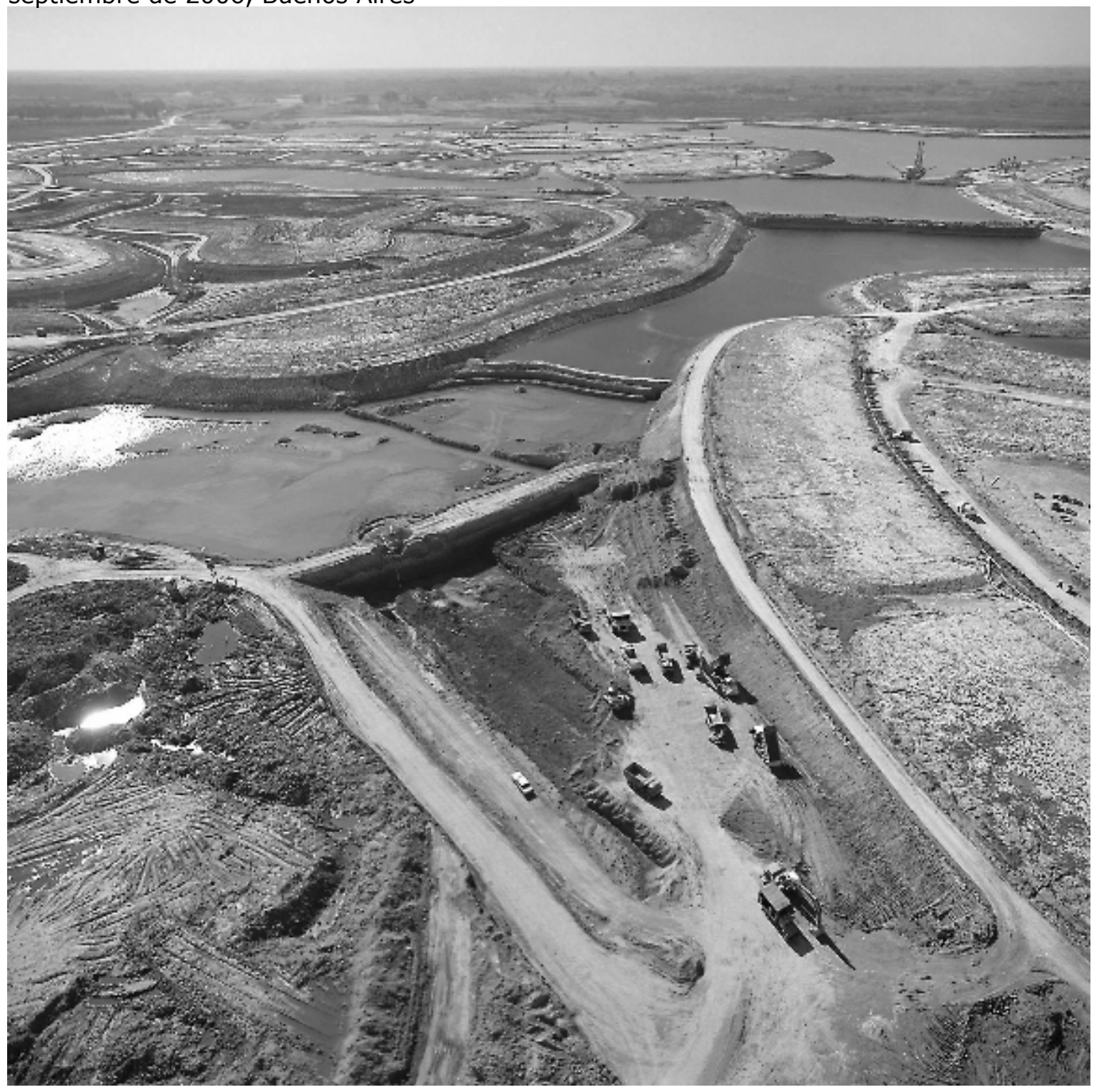

Asimismo, la elaboración de un andamiaje discursivo asociado a las nociones de "desarrollo urbano sustentable" y "valorización del paisaje" acompañó ese proceso, y en un contexto de creciente "sensibilidad ecológica", se orientó a legitimar y viabilizar el avance de esos negocios urbanos. Ese discurso fue introducido por los empresarios inmobiliarios y el propio Estado local justificando la producción de UCs sobre rellenos como alternativa sustentable para urbanizar las tierras inundables de los bañados del municipio de Tigre con productos de "alta calidad ambiental" (RÍOS y PÍREZ, 2008).

El proceso de producción de espacio urbano sobre áreas inundables y, con ello, obviamente de riesgo de desastres aquí presentado, es resultante de un conjunto de racionalidades convergentes. Por un lado, se advierte la necesidad que tiene el capital 
inmobiliario-financiero, en un momento de notoria expansión a nivel mundial (fase de globalización del capitalismo actual), de reproducirse aunque sea generando riesgo de desastres. El relleno de miles de hectáreas ${ }^{15}$ de los bañados tigrenses como "solución ingenieril" al problema de las inundaciones implica la posibilidad de una alteración de la dinámica hídrica, con peores consecuencias para los habitantes de los alrededores, e incluso de ocurrencia de alguna inundación que supere el nivel de los rellenos, perjudicando a los habitantes y a las millonarias inversiones de las UCs. A pesar de ello, también debe considerarse que la reconstrucción postdesastre se ha convertido en un redituable negocio inmobiliario-financiero, tal lo demuestra el caso de Nueva Orleáns luego del paso del huracán Katrina.

Por otro lado, es el Estado, sobre todo en los niveles de gestión Municipal y Provincial, quien genera las condiciones para que esas grandes inversiones urbanas puedan realizarse: cambios en la reglamentación, gestiones o articulación de diferentes actores públicos y privados y oferta de financiamiento, etc. Desde su perspectiva la expansión de UCs sobre los bañados involucra la posibilidad de mayores recursos para las arcas municipales, de orientar el perfil socioeconómico de Tigre hacia los sectores medio-altos (acorde con el resto de municipios de la zona norte) y de poner, al mismo tiempo, un límite al desarrollo de asentamientos precarios en esas tierras del bañado; desarrollo que se contrapone con el actual proyecto del Municipio. En suma, en nombre del "desarrollo urbano sustentable" se ensombrece que este singular proceso de producción de espacio urbano asociado a las UCs sobre rellenos del bañado de Tigre genera altos costos ambientales y sociales, como es, entre otros, la producción de riesgo de desastres; costos que se pondrán de relieve más dramática y desigualmente que nunca cuando acontezca la próxima gran inundación.

\section{Conclusiones}

En este trabajo se han presentado una perspectiva que creemos que complejiza el análisis de la producción de riesgo de desastres al incorporar en su explicación uno de los aspectos que participa de su base material: la producción de espacio. Al trabajar con dos casos del AGBA, nos remitimos a su forma urbana y a cómo fue produciéndose esa forma a lo largo de la historia: qué racionalidades dominaron, que atributos espaciales se valoraron y cuál fue el resultado en términos de producción del riesgo de desastre por inundaciones. En ambos casos se destaca cuál es el papel que ha tenido el Estado (en sus distintos niveles) como productor de espacio y productor de riesgo de desastre, fundamentalmente a partir de la reconfiguración de las relaciones público-privado, como así también el papel protagónico que han alcanzado en este proceso los actores económicos privados asociados al capital inmobiliario-financiero, a partir de la última década del siglo XX en Argentina.

En síntesis, se trata de enfatizar la necesidad de poner en primer plano el proceso histórico que explica cómo y por qué áreas donde acontecen fenómenos físicos extremos son valorizadas, apropiadas e integradas al AGBA (esta vez protagonizada por los actores hegemónicos y grupos medio-alto y altos) a través del proceso de urbanización, creando las condiciones para la construcción de situaciones de vulnerabilidad social, que se ponen en evidencia cada vez que aparece una inundación.

\section{Notas.}

1. Este trabajo constituye una versión mejorada de una clase que los autores realizaron para el curso de posgrado virtual FLACSO-Argentina: "Tratamiento de catástrofes en condiciones subdesarrolladas: riesgo, vulnerabilidad social e incertidumbre", dictado durante agosto-diciembre de 2007.
2. La propiedad abarcaba casi todo el Bañado. Rosas hizo rellenar los bajíos con tierra que traía desde otras partes, incluyendo las orillas del propio arroyo Maldonado. Además de la vivienda, construyó un canal artificial donde se practicaba el remo y la navegación a vapor (DIRECCIÓN GENERAL DE PATRIMONIO, op. cit). 
3. Las obras consistieron en el revestimiento y unión de las columnas que sostienen el techo de la canalización a través de un tabique ("entabicado") y en la remoción de un antiguo puente carretero en el cruce de la Av. Santa Fe con Juan B. Justo, que había quedado bajo el asfalto y formando parte del conducto (GONZÁLEZ, 2006).

4. Ellas son la Ley $23.686 / 89$ de Reforma del Estado, que impulsa el proceso privatizador y la Ley $23.928 / 91$ de Convertibilidad $(1$ peso $=1$ dólar).

5. El mecanismo de excepción se utilizó para construir el ejemplo paradigmático de estas torres: el edificio Le Parc, construido entre 1992 y 1995 en Palermo Nuevo. De 50 pisos de altura, su construcción se opuso a varias de las disposiciones del CPU. Puede decirse que este edificio inició el proceso de reconversión en Palermo, brevemente descrito más adelante.

6. El primer período de verticalización de la Ciudad se produjo con la sanción del Código de Edificación del año 1944 y de la Ley 13.512/48 de Propiedad Horizontal que permitieron la construcción en altura y modelaron la producción del espacio hasta el año 1977, cuando se sancionó la primera versión del CPU.

7. Son las llamadas "amenities premium": canchas de tenis y golf, piscinas climatizadas, solarium, gimnasio, spa, lavaderos, juegos para niños, etc.

8. Este contacto se expresa en la parquización (jardines de diseño) que rodea las torres.

9. Un ejemplo es el Programa de Desarrollos Estratégicos en Playas Ferroviarias del Gobierno de la Ciudad, que plantea la liberación de buena parte de terrenos afectados al nodo ferroviario Palermo.

10. Se trata de la recuperación de antiguos talleres ferroviarios y el edificio de la Bodega Giol (desalojada de las familias carenciadas que la ocuparon hasta 1994), donde se plantea el proyecto Palermo Centro, un complejo comercial y cultural.
11. En la cartografía posterior a ese evento, confeccionada por el ingeniero Eustaquio Giannini (1805) propuesta para el trazado del nuevo canal San Fernando, se indica que las aguas alcanzaron la base de la barrancas próximas, por lo que a valores actuales esa cota de inundación habría alcanzado alrededor de los $5 \mathrm{~m}$ (IGN) (RÍOS, 2010).

12. Un claro ejemplo de ello, fue la valorización de las áreas costeras de los ríos y arroyos Las Conchas/Reconquista, Luján y Tigre, por parte de la elite porteña (apoyada con importantes inversiones públicas) como sitio para la construcción de residencias de verano, clubes de remo, hoteles y casinos, que permitían la realización de prácticas deportivas y recreativas. Así, entre fines del siglo XIX y comienzos del $X X$, se configura una importante villa balnearia sobre parte del antiguo puerto de Las Conchas y de Tigre. En este caso lo grupos de la elites se ven atraídos no sólo por una valorización de una naturaleza en un sentido productivo, sino, más aún, por una naturaleza en un sentido estético, evidente en los discursos de distintas personalidades de la época, como fue el presidente D. F. Sarmiento, quien tuvo un papel destacado en la promoción del Tigre y de las islas del Delta (ver sus publicaciones como El Carapachay o Argirópolis). Aquí los réditos económicos obtenidos en este proceso le restaban valor a las consecuencias negativas vinculadas con la inundabilidad.

13. Asimismo, esta expansión se relaciona con un cambio de la intervención estatal menos orientada por los "intereses generales", que por intereses económicos particulares. Se observa en términos generales un pasaje de un Estado "administrativo" a uno "emprendedor", cuya tendencia es la conformación de coaliciones entre actores públicos y actores económicos privados para atraer mayores inversiones.

14. La Unidad de Coordinación del proyecto río Reconquista (UNIREC), ente antártico de Ministerio de Obras y Servicios Públicos de la Provincia de Buenos Aires, ha realizado obras de Saneamiento y control de inundaciones sobre esa cuenca, 
las cuales han servido para legitimar la idea de solución contra las inundaciones utilizadas tanto por los desarrolladores de las UCs como por el gobierno del Municipio de Tigre.

15. Los rellenos constituyen técnicas constructivas de adaptación para urbanizar áreas inundables y/o anegables. Estas técnicas pueden ser construidas a través de dos métodos de movimientos de suelo: uno en "seco" con maquinaria mecánica (retroexcavadoras, palas mecánicas, camiones) y el otro en "líquido" a través de refulado hidráulico con dragas. Para este último método, debe construirse endicamientos perimetrales que luego son rellenados a través de mangas con agua y suelos (refulado hidráulico). Una vez expulsados los líquidos, los suelos decantan y forman el relleno donde luego se construye "fuera del alcance" de las inundaciones. Estas técnicas implicaron un giro radical en las estrategias elaboradas para adaptarse a las condiciones físicas de inundabilidad en la historia de la urbanización de los bañados de Tigre, siendo totalmente distintas a las técnicas precedentes tales como palafitos, pequeños rellenos, polderizaciones u otras (RÍOS, 2010).

16. Las mega-UCs Nordelta con 1.643 ha. y Villa Nueva con 855 ha., representan algo más del $70 \%$ de las tierras ocupadas por las UCs sobre rellenos (RÍOS, 2010).

\section{Bibliografia}

CARLOS, A. A (re)produçao do espaço urbano. Sao Paulo: Edusp, 2008.

CLICHEVSKY, N. Política social urbana. Normativa y configuración de la ciudad. Buenos Aires: Espacio Editorial, 1996.

CLICHEVSKY, N. Pobreza y políticas urbano-ambientales en Argentina. Santiago de Chile: CEPALECLAC, División de Medio Ambiente y Asentamientos Humanos, 2002.

DIRECCIÓN GENERAL DE PATRIMONIO. Buenos Aires, Paisaje Cultural de la Humanidad UNESCO. Buenos Aires: Secretaría de Cultura del Gobierno de la Ciudad de Buenos Aires, 2004.

GARCÍA ACOSTA, V. "El riesgo como construcción social y la construcción social del riesgo", en Desacatos, Revista de Antropología Social. No 19, 2005, p. 11-24. México, CIESAS. Disponible en sitio web: <http://www.ciesas.edu.mx/Desacatos/ Ini.html >

GONZÁLEZ, S. Gestión urbana pública y desastres. Inundaciones en la baja cuenca del arroyo Maldonado (Capital Federal, 1880-1945). Tesis de Licenciatura en Geografía, Buenos Aires: FFyL/ UBA, mimeo, 1997.

GONZÁLEZ, S. "O planejamento urbano e a construção do risco por inundações na cidade de Buenos Aires", en: CARLOS A.; DE OLIVEIRA, A. (org.) Geografias das Metrópoles. São Paulo: Editora Contexto, 2006, p. 467-484.

GONZÁLEZ, S. Ciudad visible vs. ciudad invisible. Gestión urbana y manejo de inundaciones (Ciudad de Buenos Aires) en la baja cuenca del arroyo Maldonado. Tesis de doctorado, Buenos Aires: FFyL/UBA, mimeo, 2009.

HARDOY, J. "Localización y causas de abandono de las ciudades hispanoamericanas durante las primeras décadas del siglo XVI", en: HARDOY J.; MORSE, R. (comp.) Nuevas perspectivas en los estudios sobre historia urbana latinoamericana, Buenos Aires: GEL, 1989, p. 9-39.

HERZER, H; CLICHEVSKY, N. "Perspectiva histórica: las inundaciones en Buenos Aires". En: KREIMER, A. et al. (eds.), Inundaciones en el Área Metropolitana de Buenos Aires. Washington D.C.: World Bank, Disaster Management Facility, 2001, p. 33-45. Disponible en sitio web: <http://siteresources.worldbank.org/INTDISMGMT/Resources/ buenosaires.pdf $>$

LINDÓN, A. "La problemática de las inundaciones en áreas urbanas como proceso de ocupación, un enfoque espacio-temporal. El caso de la Ciudad de Buenos Aires", Actas del II Encuentro de Geógrafos 
de América Latina. Tomo I, problemática urbana. Montevideo, 1989, p. 33-43.

MANSILLA, E. Riesgo y ciudad. México: Universidad Autónoma de México, División de Estudios de Posgrado, Facultad de Arquitectura, 2000. Disponible en sitio web: <http://www.desenredando.org>

MORAES, A.; DA COSTA, W. Geografía Crítica. A valorização do espaço. São Paulo: Editora HUCITEC, 1987.

RÍOS, D.; PÍREZ, P. "Urbanizaciones cerradas en áreas inundables del municipio de Tigre: ¿producción de espacio urbano de alta calidad ambiental?". EURE, 34, 101, 2008, p. 99-119.

RÍOS, D. Producción de espacio de riesgo de desastres a partir de la urbanización de áreas inundables. Los bañados de Tigre, su historia y sus transformaciones recientes. Tesis de doctorado. Buenos Aires: FFyL/UBA, mimeo, 2010.

SILVA, H. "El comercio ilícito en el Río de la Plata", Todo es historia, 260, 1989, p. 7081.

STEINBERG, T. "The secret history of natural disaster", Environmental Hazards, 3, 2001, p. 31-35.

SZAJNBERG, D.; CORDARA, C. "La transformación de Palermo Nuevo, Pacífico y el eje de Juan B. Justo-Intendente Bullrich", Café de las Ciudades. Conocimiento, reflexiones y miradas sobre la ciudad. Revista electrónica. Año 4, No 35, 2005. Disponible en sitio web: <http://www.cafedelasciudades.com>

UDAONDO, E. Reseña histórica del Partido de Las Conchas, Tigre: Municipalidad de Tigre, 2001. 